\title{
Magnetic instability with increasing hybridization in cerium compounds
}

\author{
Nicholas Kioussis \\ Department of Physics, California State University, Northridge, Northridge, California 91330 \\ Bernard R. Cooper \\ Department of Physics, West Virginia University, Morgantown, West Virginia 26506
}

John M. Wills

Los Alamos National Laboratory, Los Alamos, New Mexico 87544

(Received 6 March 1991)

\begin{abstract}
A synthesis of a phenomenological theory of orbitally driven magnetic ordering of moderately delocalized light rare-earth systems and ab initio electronic structure calculations has been applied to investigate the change in magnetic behavior on going from $\mathrm{CeSb}$ to $\mathrm{CeTe}$, both of which have rocksalt structure with a small decrease in lattice parameter. The hybridization-potential matrix elements and the band energies entering the Anderson-lattice Hamiltonian are obtained from linear-muffin-tin-orbital (LMTO) electronic-structure calculations with the Ce $4 f$ states treated as core states. The position of the Ce $4 f$ energy level relative to the Fermi energy and the intra-atomic Coulomb energy $U$ are obtained by use of a sequence of three total-energy supercell calculations with one out of four Ce sites constrained to $f^{n}$ occupation with $n=0,1,2$, successively. The calculations elucidate the origins, in the electronic structure, of the variation of the $f$-state resonance width and hybridization potential on going from $\mathrm{CeSb}$ to $\mathrm{CeTe}$, and the resultant sensitivity of the hybridization dressing of the crystal-field splitting and the hybridization-induced exchange interactions to chemical environment. The effect of opening up successive angular momentum scattering channels of the ab initio calculated two-ion exchange-interaction matrix on the nature of the magnetic ordering is examined. The calculated magnitude and range dependence of the two-ion exchange interactions changes sharply from $\mathrm{CeSb}$ to $\mathrm{CeTe}$, yielding a change in magnetic behavior in qualitative agreement with experiment. The nonlinear hybridization effects on the hybridization dressing of the crystal-field splitting have been examined. These effects, which are associated with the self-consistent determination of both the band states and $f$ states in the presence of band- $f$ hybridization, are found to be small in both systems.
\end{abstract}

\section{INTRODUCTION}

Among the cerium compounds, the heavier monopnictides $^{1,2} \mathrm{Ce} X(X=\mathrm{As}, \mathrm{Sb}, \mathrm{Bi})$ and monochalcogenides ${ }^{3,4}$ $(X=\mathrm{S}, \mathrm{Se}, \mathrm{Te})$ of cerium, which form in the rock salt structure, provide a class of compounds exhibiting extremely unusual magnetic properties. Of central interest are (1) the complex magnetic structures characterized by extremely strong magnetic anisotropy, ${ }^{1-5}$ (2) the large reduction of the crystal-field (CF) splitting of the $4 f_{5 / 2}$ manifold from the value expected by smooth extrapolation from the heavier rare-earth compounds; ${ }^{6}$ (3) the unusual magnetic-excitation spectra, ${ }^{7}$ and (4) great sensitivity of these properties to any perturbation such as pressure, ${ }^{8}$ the dilution 9 of cerium by nonmagnetic $\mathrm{Y}$ or La, or the substitution of a pnictogen by another pnictogen ${ }^{10}$ (Bi,As), or by a chalcogen, ${ }^{8,11}$ indicating the delicate sensitivity of hybridization-mediated exchange interactions and crystal-field effects to chemical environment.

There is a great change $\mathrm{e}^{1-4,8}$ in the nature of magnetic ordering on going from $\mathrm{CeSb}$ to $\mathrm{CeTe}$ with a small decrease in lattice constant, and where the only obvious significant change is the addition of an anion $p$ electron. This is an interesting example of the way in which hybridization of partially delocalized $f$ electrons gives rise to unusual magnetic properties, which show great chemical sensitivity. Among these compounds CeSb exhibits the most complex magnetic phase diagram, containing at least 14 different magnetic structures for $H<70 \mathrm{kOe}$. $^{1,8,12}$ $\mathrm{CeSb}$ orders with a first-order transition at $T_{N} \simeq 16.2 \mathrm{~K}$ and undergoes six additional first-order phase transitions in zero field. ${ }^{1}$ The most striking feature of its low-field diagram is the occurrence of high-temperature $(8.5<T<16.2 \mathrm{~K})$ phases consisting of a periodic stacking of nonmagnetic and ferromagnetically ordered (001) planes with up or down magnetic moments along the [001] cube edge, which are close to saturation $\left(\sim 2.1 \mu_{B}\right)^{1,8,12}$ The nonmagnetic planes, which are actually paramagnetic in nature, ${ }^{8}$ disappear below $8.5 \mathrm{~K}$, yielding a type-IA antiferromagnetic structure $(\uparrow \uparrow \downarrow \downarrow)$. On the other hand, CeTe orders ${ }^{3,4,13}$ at $T_{N} \sim 2.2 \mathrm{~K}$ into a type-II antiferromagnetic structure with a very small ordered moment of only $0.2 \mu_{B}$ aligned along the $\langle 111\rangle$ direction, in sharp contrast with CeSb. Magnetization, ${ }^{8,11}$ neutron-scattering, ${ }^{8}$ and resistivity ${ }^{14}$ experiments on $\mathrm{CeSb}_{1-x} \mathrm{Te}_{x}$ have shown that for a $\mathrm{Te}$ concentration as small as $x=0.3$, both the Néel temperature and the low-temperature moment drop abruptly from $16 \mathrm{~K}$ to less than $4 \mathrm{~K}$ and from $2.1 \mu_{B}$ to $0.7 \mu_{B}$, respectively, and the nonmagnetic planes are suppressed. Moreover, the 
crystal-field splitting between the ground-state $\Gamma_{7}$ doublet and the $\Gamma_{8}$ quartet of the $\mathrm{Ce}^{3+}$ free-ion $J=5 / 2$ multiplet, which is about $37 \mathrm{~K}$ in $\mathrm{CeSb}$, increases with tellurium concentration, reaching its maximum value of about 105 $\mathrm{K}$ at $x=0.5$, and then decreases monotonically with further increase of $x$ to the value of about $32 \mathrm{~K}$ in CeTe. Thus, the CF splitting in CeTe is about the same as in $\mathrm{CeSb}$, a rather surprising result in view of the additional valence electron on the chalcogen ion.

The purpose of the work reported here was to employ the unified phenomenological/electronic-structure theory developed recently ${ }^{2,15-25}$ to investigate the origins of the variation of the hybridization-induced anisotropic interaction responsible for the dramatic change in the nature of the magnetic ordering on going from the "wellordered" weakly hybridizing CeSb to the presumably more strongly hybridizing $\mathrm{CeTe}$, which, being at the borderline between a magnetic and a nonmagnetic state, has sometimes been referred to $^{8}$ as a dense Kondo system. Remarkable success has been achieved ${ }^{2,15-22}$ in recent years in understanding the unusual magnetic properties of a class of cerium and of light actinides (U,Pu) compounds, on the basis of a phenomenological theory of orbitally driven magnetic ordering of moderately delocalized $f$ electrons hybridizing with band electrons of non- $f$ atomic parentage. The cooperative hybridization of a pair of $f$-electron ions with band electrons gives rise to a highly anisotropic interionic exchange interaction, but where the interaction with the band electrons is through the orbital rather than the spin part of the $f$-electron moments. ${ }^{20}$ Initially, the phenomenological hybridizationmediated two-ion parameters entering the theory were taken as ${ }^{2,15-22}$ adjustable parameters to fit the observed Néel temperature, low-temperature moment, and excitation spectrum. This empirical evaluation of the phenomenological parameters did little to elucidate the origins, in the electronic structure, of the observed behavior and lacked predictive power in extending the theory to other systems. Striking changes in observed phenomena often occur for relatively small changes in electronic structure. Recently Wills and Cooper ${ }^{23}$ have developed a theory and computational technique to synthesize information from first-principles electronic-structure calculations into a phenomenological theory to make it material predictive. The resultant theory has been successfully applied ${ }^{23-25}$ to the cerium monopnictide series, yielding results for the crystal-field splitting in excellent agreement with experiment. ${ }^{6}$ On the other hand, the $p-f$ mixing model introduced by Takahashi and Kasuya ${ }^{26}$ for the cerium monopnictides is based on a simple tight-binding model, neglects the $d-f$ hybridization and considers for simplicity the $p-f$ hybridization only at the $\Gamma$ point.

In Sec. II we review briefly and generalize the phenomenological model of hybridization-mediated interactions by including all elements of the exchange two-ion interaction matrix. The calculation of the bands, the band- $f$ hybridization matrix elements, the position of the $f$ state, and the $f-f$ intraatomic Coulomb interaction $U$ is described in Sec. III. Numerical results of the linearmuffin-tin-orbital (LMTO) electronic-structure calculations, the Anderson-model parameters, the hybridization-dressing of the crystal-field splitting, the hybridization-mediated two-ion exchange interaction parameters, and the magnetic equilibrium behavior of $\mathrm{CeSb}$ and CeTe are presented and compared in Sec. IV. The importance of nonlinear hybridization effects, associated with the self-consistent determination of both the band states and the $f$ states in the presence of the band- $f$ hybridization, on the hybridization-dressing of the crystalfield splitting are presented in Sec. V. Finally, a brief summary and statement of conclusions are presented in Sec. VI.

\section{MODEL HAMILTONIAN}

The treatment of hybridization between the quasilocalized $f$ electrons and band electrons was developed by Coqblin and Schrieffer ${ }^{27}$ for isolated cerium impurity ions, and was subsequently extended by Siemann and Cooper ${ }^{15}$ to treat the hybridization-mediated anisotropic two-ion coupling. Here briefly we review the model. The phenomenological theory is based on the Anderson Hamiltonian for a lattice of $f^{1}$-electron ions hybridizing with a sea of band electrons. ${ }^{2,15-23}$ The Hamiltonian consists of terms for band and localized $f$ energies $\left(\varepsilon_{\mathbf{k}}\right.$ and $\left.E_{m}\right)$ with a correlation energy cost $(U)$ to add or subtract an $f$ electron from the nominal $f$ configuration of the ions, and a hybridization term that mixes band and $f$ electrons. Upon applying the Schrieffer-Wolff ${ }^{28}$ transformation to fourth-order in the band- $f$ hybridization-potential matrix element $\left(V_{\mathrm{k} m}\right)$, the hybridization is transformed to resonant scattering of band electrons off $f$ electrons, which is equivalent to a virtual configuration transition for the $f$-electron ion. ${ }^{2,24}$ This gives rise to the following. 2,23

(a) A shift of the crystal-field energy levels of the $4 f_{5 / 2}$ state multiplet (hybridization dressing of the crystal field) of the form ${ }^{23}$

$$
\begin{aligned}
\delta E_{M}= & \sum_{\varepsilon_{\mathbf{k}}>E_{F}} J(\mathbf{k}, M, 0 ; \mathbf{k}, M, 0)+\sum_{\varepsilon_{\mathbf{k}}<E_{F}} J(\mathbf{k}, M, 1 ; \mathbf{k}, M, 1) \\
& -\sum_{\varepsilon_{\mathbf{k}}<E_{F}} \sum_{M^{\prime}} J\left(\mathbf{k}, M^{\prime}, 1 ; \mathbf{k}, M^{\prime}, 1\right)
\end{aligned}
$$

where

$$
J(\mathbf{k}, M, n ; \mathbf{k}, M, n)=-\left|V_{\mathbf{k} M}\right|^{2} /\left(\varepsilon_{\mathbf{k}}-E_{M}-n U\right) .
$$

Here, $n$ labels the nominal $f$-ion configuration, and $M$ denotes the CF states $\left(\Gamma_{7}\right.$ or $\left.\Gamma_{8}\right)$. The parameter $J$ which is second order in the hybridization, induces at zero temperature virtual transitions between the initial and final states with one $\left(n_{f}=1\right) f$ electron per Ce site, involving only the $4 f^{0}$ and $4 f^{2}$ configurations as virtual intermediate states of energies $E_{f}-E_{F}$ and $E_{f}-E_{F}+U$, respectively. The band energies $\varepsilon_{\mathbf{k}}$ and the localized energy $E_{M}=E_{F}-E_{f}$ in Eq. (2) are both measured relative to the Fermi energy. ${ }^{27}$ However, the energies $E_{M}$ and $U$ characterizing the virtual excitation of electrons and holes from the band onto the $f$ level, are not one-electron energies, and they have to be calculated from selfconsistent total-energy supercell electronic-structure calculations as is described in detail in Sec. III. Note that 
the first term in Eq. (1), which is negative and is the dominant term for large $U$, couples the $f^{0}$ state below the Fermi energy with band states above the Fermi energy; the second term, which was neglected by Takahashi and Kasuya, ${ }^{26}$ is positive, reduced by a factor of $\approx 1 / U$, and couples the $f^{1}$ state above the Fermi energy with band states below the Fermi energy; and the last term yields a uniform lowering of all the CF energy levels.

(b) An indirect orbitally driven anisotropic two-ion exchange interaction via correlated configuration transitions of two $f$-electron ions (two-ion interaction via cooperative hybridization with the band sea). ${ }^{2,23}$

Upon transforming the $\mathrm{Ce}^{3+}$ ions $f$ states $\left|J M_{J}\right\rangle$ (which are quantized with respect to the interionic axis) to states $|\mu\rangle$ quantized with respect to one common crystal-lattice axis and summing over all $f$-electron ions, the hybridization-mediated exchange Hamiltonian assumes the form

$H=-\sum_{i, j} \sum_{\mu, v} \sum_{\varepsilon, \sigma} \mathfrak{B}_{\mu \nu}^{\varepsilon \sigma}\left(\theta_{i j}\right) e^{-i(\mu-v+\varepsilon-\sigma) \varphi_{i j}} L_{\mu \nu}^{i} L_{\varepsilon \sigma}^{j}$,

where

$$
\begin{aligned}
\mathfrak{B}_{\mu \nu}^{\varepsilon \sigma}(\theta)=\sum_{m_{1}, m_{1}^{\prime}} \sum_{m_{2}, m_{2}^{\prime}} & E\left(m_{1}, m_{1}^{\prime} ; m_{2} m_{2}^{\prime} ; \mathbf{R}\right) \\
& \times\left[d_{\mu m_{1}^{\prime}}^{(J)}(\theta) d_{v m_{1}}^{(J)}(\theta)-(2 J+1)^{-1} \delta_{m_{1} m_{1}^{\prime}} \delta_{\mu \nu}\right]\left[d_{\varepsilon m_{2}^{\prime}}^{(J)}(\theta) d_{\sigma m_{2}}^{(J)}(\theta)-(2 J+1)^{-1} \delta_{m_{2} m_{2}^{\prime}} \delta_{\varepsilon \sigma}\right] .
\end{aligned}
$$

Here, $i$ and $j$ label cerium sites, $\theta_{i j}$ and $\phi_{i j}$ are the angular coordinates of the interionic axis $\mathbf{R}_{i j}$ with respect to the axis of quantization chosen along the [001] direction in the crystal, $\mu, v, \varepsilon, \sigma$ label the single-ion states of the $z$ component of the angular momentum $\left(J=\frac{5}{2}\right)$ quantized along the [001] direction. The standard basis operators, ${ }^{29} L_{\mu \nu}^{i} \equiv|\mu\rangle\langle\nu|$ transfer the $\mathrm{Ce}^{3+}$ ion on site $i$ from state $|v\rangle$ to state $|\mu\rangle, d_{\mu m}^{(J)}$ are the matrix elements of the angular momentum rotation matrix, ${ }^{30}$ and $E\left(m_{1}, m_{1}^{\prime} ; m_{2}, m_{2}^{\prime} ; \mathbf{R}\right)$ are the hybridization-mediated two-ion exchange matrix elements given by Eqs. (2.7) and (2.8) in Ref. 23.

To study the equilibrium magnetic behavior of a given magnetic structure, we treat the Hamiltonian of Eq. (3) within mean-field theory, diagonalize the resultant expression, determine the molecular-field eigenvectors and eigenvalues for each sublattice of the magnetic structure considered, repeating the procedure iteratively until selfconsistency is achieved. ${ }^{2,15-22}$ For a given neighbor distance, the exchange interaction matrix $E(R)$ has $6^{4} / 4$ independent components (reduction by a factor of 4 comes from time-reversal symmetry). ${ }^{24}$ Thus, in practice, one has to reduce the size of the two-ion exchange matrix to obtain initial physical understanding before inserting the full complexity into the absolute theory for the phenomenology. For the simple case of azimuthal symmetry about the interionic axis $\mathbf{R}$, which is the case for freeelectron bands, the angular-momentum component along $\mathbf{R}$ is conserved by the two-ion interaction in Eq. $(3) ; 2,23,24$ hence, with $f$ states $|m\rangle$ quantized with respect to $\mathbf{R}, m_{2}=m_{1}^{\prime}$ and $m_{2}^{\prime}=m_{1}$, and the number of independent parameters for the $\mathrm{Ce}^{3+}$ lattice is reduced to nine. Furthermore, it may be shown ${ }^{2}$ that the axial symmetry of the free-electron bands about $\mathbf{R}$ implies that, in the limit $k_{F} R \rightarrow \infty$, the predominant components of $E(\mathbf{R})$ are those for which $m_{1}, m_{1}^{\prime}= \pm 1 / 2$ with respect to $\mathbf{R}$. These involve the $m_{l}=0$ part of the $f$ wave function, corresponding to the piling up of charge along the interionic axis. Thus, in this limit a single exchange parameter $E_{n}$ is required for each symmetry shell $n,{ }^{2,23,24}$ and the matrix elements of $\mathfrak{B}(\theta)$ assume the simple form

$$
\mathfrak{B}_{\mu \nu}^{\varepsilon \sigma}\left(\theta_{i j}\right)=E_{i j} B_{\mu \nu}^{\varepsilon \sigma}\left(\theta_{i j}\right)
$$

i.e., they separate into a product of the range parameter $E_{i j}$ which depends only on the symmetry shell, and the purely geometric quantity $\boldsymbol{B}(\theta)$ whose angular dependence is the same for every symmetry shell, of the form, ${ }^{2}$

$$
B_{\mu \nu}^{\varepsilon \sigma}(\theta)=\sum_{m_{1}, m_{1}^{\prime}= \pm 1 / 2}\left[d_{\mu m_{1}^{\prime}}^{(J)}(\theta) d_{v m_{1}}^{(J)}(\theta)-(2 J+1)^{-1} \delta_{m_{1} m_{1}^{\prime}} \delta_{\mu \nu}\right]\left[d_{\varepsilon m_{1}}^{(J)}(\theta) d_{\sigma m_{1}^{\prime}}^{(J)}(\theta)-(2 J+1)^{-1} \delta_{m_{1} m_{1}^{\prime}} \delta_{\varepsilon \sigma}\right]
$$

It is important to note that the angular dependence of the anisotropic exchange interaction [Eq. (4)] for the general case does depend upon the symmetry shell. The parameters $E_{n}$ with $n \leq 3$ were initially taken ${ }^{2,15-22}$ as adjustable parameters to fit the observed Néel temperature, zerotemperature moment, and low-temperature excitation spectrum.

\section{AB INITIO EVALUATION OF MODEL HAMILTONIAN PARAMETERS}

In this section we briefly review the theory and computational technique, recently developed ${ }^{23-25}$ to synthesize first-principles electronic structure information into the phenomenological theory ${ }^{2,15-22}$ discussed above to make it material predictive. The electronic structure calculations, based on the linear-muffin-tin-orbital (LMTO) method, ${ }^{31}$ allow an $a b$ initio evaluation of the parameters entering the phenomenological theory. These are the band energies $\varepsilon_{n \mathbf{k}}$, the position of the $f$-level $E_{f}$ with respect to the Fermi energy, the intra-atomic $f$ - $f$ correlation energy $U$, and the hybridization potential matrix elements $V_{\mathbf{k} m}$ between band states and $f$ states.

The first step is the generation of a self-consistent oneelectron potential describing the band structure of the compound of interest. This is obtained from a warped- 
muffin-tin LMTO band-structure calculation, ${ }^{23}$ i.e., a LMTO calculation in which the potential is spherical (except for a small correction) in nonoverlapping spheres and has unrestricted spatial dependence in the interstitial. This calculation differs from the more common LMTO-atomic-sphere-approximation method ${ }^{31}$ in that the LMTO bases have, in general, a nonzero kinetic energy (tail parameter or energy window), $\kappa^{2}$, in the interstitial, which is treated as a variational parameter. Three energy windows are employed to allow coverage of various subbands: the semicore cerium $5 p$ and the pnictogen or chalcogen $5 s$ states are calculated with a tail parameter about $-1 \mathrm{Ry}$ and $-0.3 \mathrm{Ry}$, respectively, and the cerium valence $6 s, 6 p$, and $5 d$ states and pnictogen or chalcogen $5 p$ states with a tail parameter, which is the average energy over occupied states in the interstitial. The electron density and potential in the interstitial region are expanded in Fourier series. Exchange and correlation are treated in the Hedin-Lundqvist ${ }^{32}$ version of the local-density approximation. The potential is evaluated at the experimental volume, and the radii of the nonoverlapping muffin-tin spheres are chosen to make the potential continuous where spheres touch.

The $4 f^{1}$ states in the Anderson model Hamiltonian ${ }^{27}$ are localized on cerium sites and do not couple to band states of non- $f$ atomic parentage. Consequently, in calculating the self-consistent potential for the compound, the cerium $f$ states are treated ${ }^{23}$ as localized rather than itinerant states, and they are included self-consistently as core states at each iteration, not being allowed to hybridize with band states. Thus at each iteration the $f$ states are treated as resonant states that are constrained to be localized. ${ }^{23,24}$ The core states are obtained from the Dirac equation for the spherically averaged potential at each site and are included self-consistently, i.e., no "frozen" core approximation is made. The radial basis function for the (non- $f$ ) bands within the muffin-tin spheres are solutions of the scalar relativistic radial equation. ${ }^{33}$ Spin-orbit coupling is included self-consistently.

The electronic-structure calculation provides us with a self-consistent potential with which one calculates the band energies and hybridization matrix elements entering the model Hamiltonian. Because accurate determination of these parameters requires a highly accurate representation of the bands (cerium-derived $d$ bands and anionderived $p$ bands) around the Fermi energy, one needs to perform a final band calculation, after the selfconsistency process, with the tail parameter of the highest-energy window set equal to the Fermi energy. The hybridization potential matrix elements assume the form ${ }^{23}$

$$
V_{n \mathbf{k}, m}=v\left(\kappa_{F}\right) \sqrt{\Omega}\left[T_{m}^{(n)}(\mathbf{k})\right]^{*},
$$

where $\kappa_{F}^{2}=E_{F}, T_{m}^{(n)}(\mathbf{k})$ is given in terms of products of the Korringa-Kohn-Rostoker structure-function matrix and the LMTO eigenvectors, and

$$
v\left(\kappa_{F}\right)=-\left(\frac{\Gamma}{2 \kappa_{F} N \Omega}\right)^{1 / 2}
$$

is the hybridization potential. ${ }^{23}$ Here, $\Omega$ is the unit cell volume, and the resonance width is given by ${ }^{23}$

$$
\Gamma=\frac{2}{\kappa_{F}}\left(\frac{\varphi_{f}(s)}{h_{3}^{+}\left(\kappa_{F} s\right)}\right)^{2},
$$

where $\varphi(s)$ is the resonant radial $f$ wave function at the cerium muffin-tin radius $s$, and $h_{3}^{+}$is the $l=3$ spherical Hankel function of the first kind.

The energy necessary to place a cerium $f$ electron in a band state at the Fermi energy $E_{F}-E_{f}$ and the energy required to change from an $f^{1}$ to an $f^{2}$ configuration, $E_{f}+U-E_{F}$, are obtained from self-consistent localdensity supercell band-structure calculations, in which one considers eight atoms per unit cell as opposed to two for the ground-state calculation. The atoms consist of a central $\mathrm{Ce}$ site $\mathrm{Ce}(1)$, three other equivalent $\mathrm{Ce}$ sites $\mathrm{Ce}(2)$, three equivalent pnictogen or chalcogen sites $X(1)$, which border $\mathrm{Ce}(1)$, and another inequivalent pnictogen site $X(2)$ ( $X$ denotes the pnictogen or chalcogen site). These energies are obtained from self-consistent LMTO total-energy calculations with constrained $\mathrm{Ce}\left(4 f^{n}\right)$ impurity-site occupation $n$, where one removes (adds) an $f$ electron to the central cerium site $\mathrm{Ce}(1)$ and allows the system to relax, resulting in a $d$ screening (antiscreening) of the $f$ hole (electron). ${ }^{34}$ Using the property of the local-density-functional approximation that the eigenvalue is the occupation-number derivative of the total energy, the difference in the total energy between the initial and final states can be written as ${ }^{34}$

$$
\Delta E=-\int_{0}^{1}(\delta E / \delta n) d n=-\int_{0}^{1} \varepsilon_{f}^{(n)} d n,
$$

where $n=0,1$ is the occupation number of the central $\mathrm{Ce}(1)$ site in the initial and final states, and $\varepsilon_{f}^{(n)}$ is the selfconsistent one-electron eigenvalue of the $4 f^{n}$ configuration of the $4 f$ state of the central $\mathrm{Ce}(1)$ site, which is treated as a core state rather than as an itinerant state, thus not being allowed to hybridize with band states. Because to a good approximation the eigenvalue is a linear function of the occupation, the value of the integral is equal to the average of the end points, ${ }^{34}$

$$
E_{F}-E_{f}=-\left(\varepsilon_{f}^{(1)}+\varepsilon_{f}^{(0)}\right) / 2 \text {. }
$$

Similarly one finds that

$$
E_{F}-E_{f}-U=-\left(\varepsilon_{f}^{(1)}+\varepsilon_{f}^{(2)}\right) / 2 .
$$

The energy $E_{M}=E_{F}-E_{f}$ in Eq. (2) is the total energy required to remove a $\mathrm{Ce}^{3+} f$ electron and place the electron at the Fermi energy ( $f^{1} \rightarrow f^{0}$ transition) with all other electrons readjusting to screen the $4 f$ hole. ${ }^{27,34}$ In other words, this energy represents the energy difference between the ground state and a system consisting of one of the $\mathrm{Ce}$ ions in the $4 f^{0}$ configuration plus one electron at the Fermi energy. The ground state consists of $N \mathrm{Ce}$ ions that are in a well-defined level with total angular momentum $J=5 / 2$, which derives from a given term of the $4 f^{1}$ configuration and an arbitrary number of conduction electrons (conduction-electron reservoir). Similarly, $E_{f}+U-E_{F}$ is the total energy required to add an electron to the ground-state configuration $\left(4 f^{1}\right)$ of one of the $\mathrm{Ce}^{3+}$ impurities from the Fermi level ( $f^{1} \rightarrow f^{2}$ transition) 
with all other electrons readjusting to antiscreen the $4 f$ electron. Again, this energy represents the energy difference between the $4 f^{2}$ configuration and the system consisting of an ion in the ground state and a band electron at the Fermi energy. Thus, both these energies, which appear in Eq. (2), are the energies characterizing the virtual excitations of electrons and holes from the band onto the $f$ level. However, both these energies are not one-electron energies as is the resonance energy $\varepsilon_{f}^{(1)}$ of the $4 f^{1}$ corelike state, and they have to be calculated from Eqs. (11) and (12), respectively. ${ }^{34}$

Having determined the model parameters, the crystalfield splitting and the magnetic phase diagram can be calculated from Eqs. (1) and (3), respectively. The kdependent quantities $\varepsilon_{\mathbf{k}}, V_{\mathrm{k} m}$, and the total and muffintin sphere $l$-projected density of states are calculated on a tetrahedral mesh, ${ }^{35}$ using 89 points in the irreducible portion of the fcc Brillouin zone.

\section{RESULTS AND DISCUSSION}

\section{A. Electronic structure}

The band structures of $\mathrm{CeTe}$ and $\mathrm{CeSb}$ are plotted along symmetry lines in Figs. 1 and 2, with the band energies measured with respect to $E_{F}$. Also shown in Figs. 1 and 2 is the symmetry of the bands at $\Gamma$. Anion $p$ derived bands at $\Gamma$ have $\Gamma_{6}^{(-)}$and $\Gamma_{8}^{(-)}$symmetry, while cerium $5 d$-derived bands at $\Gamma$ are the bases for three representations, one with $\Gamma_{7}^{(+)}$and two with $\Gamma_{8}^{(+)}$symmetry. The bands dominating the hybridization-induced properties are largely derived from anion $p$ states and cerium $5 d$ states. It is important to note that, on going from $\mathrm{CeSb}$ to $\mathrm{CeTe}$, the addition of an anion $p$ electron has caused the $p$-derived bands to sink far below the Fermi energy, thus suppressing the $p-f$ hybridization. Consequently, the bands dominating the hybridization-induced proper-

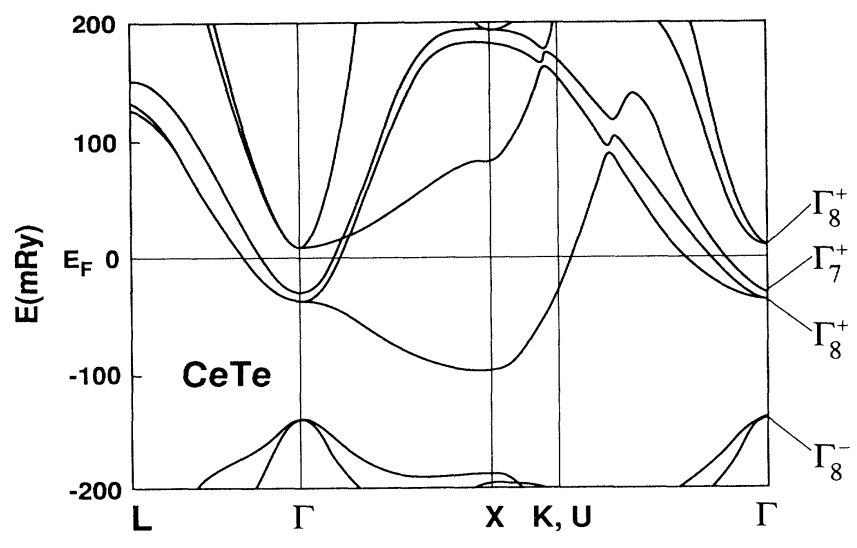

FIG. 1. The band structure of CeTe, calculated with the Ce $4 f$ states treated as core states, along symmetry lines in the Brillouin zone. The band energies are with respect to the Fermi energy. The labels on the right of the figure denote the symmetry of the bands at $\Gamma$. Anion $p$-derived bands at $\Gamma$ have $\Gamma_{8}^{(-)}$symmetry, while Ce $5 d$-derived bands have $\Gamma_{7}^{(+)}$or $\Gamma_{8}^{(+)}$symmetry.

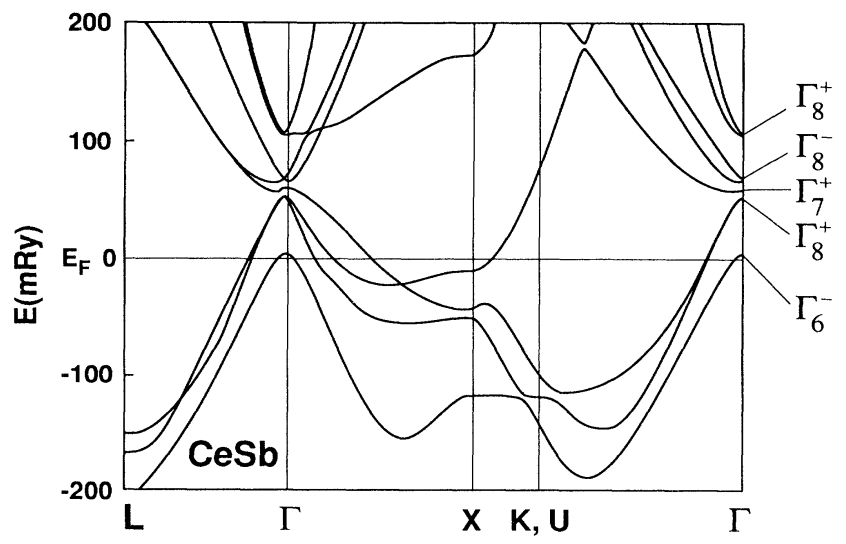

FIG. 2. The band structure of $\mathrm{CeSb}$, calculated with the $\mathrm{Ce}$ $4 f$ states treated as core states, along symmetry lines in the Brillouin zone. The band energies are with respect to the Fermi energy. The labels on the right of the figure denote the symmetry of the bands at $\Gamma$. Anion $p$-derived bands at $\Gamma$ have $\Gamma_{6}^{(-)}$or $\Gamma_{8}^{(-)}$symmetry, while Ce $5 d$-derived bands have $\Gamma_{7}^{(+)}$or $\Gamma_{8}^{(+)}$ symmetry.

ties in CeTe are largely derived from the Ce $5 d$ states. The energy of the resonant corelike cerium $4 f_{5 / 2}$ state lies $8 \mathrm{mRy}$ and $73 \mathrm{mRy}$ below the Fermi energy in CeSb and $\mathrm{CeTe}$, respectively.

The total density of states (solid curves) of CeTe and $\mathrm{CeSb}$ corresponding to the band structures in Figs. 1 and 2 are shown in Figs. 3 and 4, respectively. Also plotted in the same figure are the partial density of states for the $\mathrm{Ce}$ $d$-derived (dotted curve) and the anion ( $\mathrm{Te}$ or $\mathrm{Sb}$ ) $p$ derived (dashed curve) bands, respectively. The Fermi energy $E_{F}$, and the density of states (DOS) at the Fermi energy $N\left(E_{F}\right)$, in CeTe are $0.482 \mathrm{Ry}$ and 15.4 states/Ry, respectively, compared to the corresponding values of

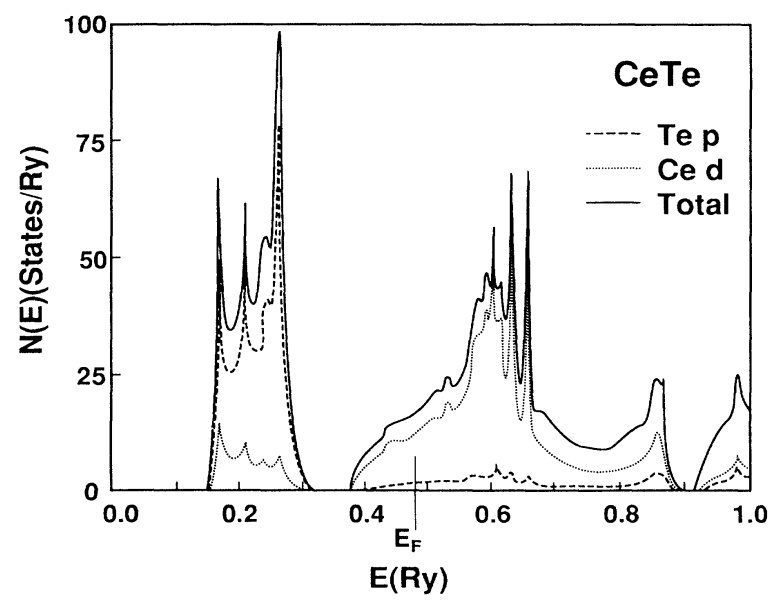

FIG. 3. The density of states of CeTe corresponding to the band structure shown in Fig. 1. Also shown are the partial density of states for the Ce $d$-derived (dotted curve) and Te $p$ derived (dashed curve) bands, respectively. 
TABLE I. Summary of LMTO results for the Fermi energy $E_{F}$, the Ce $4 f_{5 / 2}$ resonance energy, the total density of states $N\left(E_{F}\right)$ at $E_{F}$, the Ce $d$-derived and anion (Sb or Te) p-derived partial density of states at $E_{F}$, the Ce $4 f_{5 / 2}$ resonance width $\Gamma$, and the hybridization potential $v(\kappa)$, for CeSb and CeTe, respectively. Also listed are the results of the supercell LMTO calculations for the position of the $f$ state relative to $E_{F}$, and the intraatomic Coulomb interaction $U$.

\begin{tabular}{lcr}
\hline \hline & $\mathrm{CeSb}$ & CeTe \\
\hline Lattice constant (a.u.) & 12.118 & 12.020 \\
Ce muffin-tin radius (a.u.) & 3.095 & 2.837 \\
Anion muffin-tin radius (a.u.) & 2.964 & 3.173 \\
$E_{F}(\mathrm{Ry})$ & 0.376 & 0.482 \\
$\mathrm{Ce} 4 f$ resonance energy $(\mathrm{Ry})$ & 0.363 & 0.409 \\
$N\left(E_{F}\right)\left(\mathrm{Ry}^{-1}\right)$ & 4.4 & 15.4 \\
$\mathrm{Ce} d$-derived DOS at $E_{F}\left(\mathrm{Ry}^{-1}\right)$ & 1.4 & 10.3 \\
Anion $p$-derived DOS at $E_{F}\left(\mathrm{Ry}^{-1}\right)$ & 2.5 & 2.1 \\
$\Gamma(\mathrm{m} \mathbf{R})$ & 2.96 & 5.67 \\
$v(\kappa)(\mathrm{mRy})$ & -2.33 & -3.08 \\
$E_{f}-E_{F}(\mathrm{eV})$ & $-3.35^{\mathrm{a}}$ & -3.00 \\
$U(\mathrm{eV})$ & $6.1^{\mathrm{a}}$ & 6.3 \\
\hline \hline
\end{tabular}

${ }^{\text {a }}$ Reference 37.

$0.376 \mathrm{Ry}$ and 4.4 states/ $\mathrm{Ry}$ in $\mathrm{CeSb}$, indicating the metallic and semimetallic character of these compounds, respectively. Calculated results for the total and partial density of states at $E_{F}$ are listed in Table I. The increase of $N\left(E_{F}\right)$ ongoing from $\mathrm{CeSb}$ to CeTe is largely due to the increase of the $d$ density of states at the Fermi energy from 1.4 states/Ry to 10.3 states/Ry. It is exactly this large net increase in hybridization, due to the large increase in band states available for hybridization, that is responsible for the change in the range, size, and sign of the hybridization-mediated two-ion exchange interactions on going from $\mathrm{CeSb}$ to $\mathrm{CeTe}$. However, aside from the location of $E_{F}$ and the consequent density of states at $E_{F}$, and the opening of a gap in the density of states in CeTe

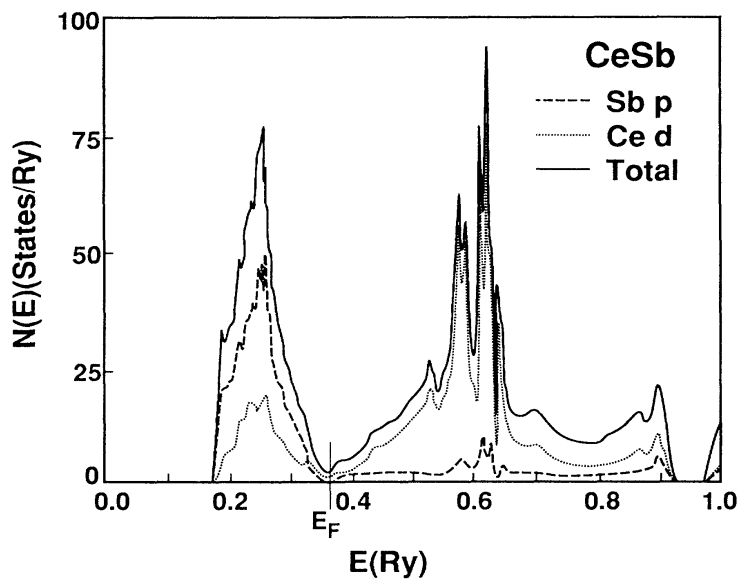

FIG. 4. The density of states of CeSb corresponding to the band structure shown in Fig. 2. Also shown are the partial density of states for the $\mathrm{Ce} d$-derived (dotted curve) and $\mathrm{Sb} p$ derived (dashed curve) bands, respectively. below $E_{F}$ between 0.325 and $0.375 \mathrm{Ry}$, the density of states for the two compounds are qualitatively similar.

\section{B. Model Hamiltonian parameters}

On going from CeSb to $\mathrm{CeTe}$ the cerium $4 f_{5 / 2}$ resonance width $\Gamma$ increases from 2.96 to $5.67 \mathrm{mRy}$, and the hybridization potential changes from $-2.33 \mathrm{mRy}$ to $-3.08 \mathrm{mRy}$, respectively, due to the increase of the hybridization of the $f$ state with the cerium $5 d$ band states. From the supercell band-structure calculation for CeTe, we find that the $4 f^{1}$ level lies $3.0 \mathrm{eV}$ below the Fermi energy in good agreement with the value of $2.6 \pm 0.1 \mathrm{eV}$ found in x-ray photoemission experiments, ${ }^{36}$ and $U=6.3$ eV. Similar results for $E_{f}-E_{F}$ and $U$, values of which are listed in Table I, have been found for CeSb. ${ }^{37}$ The angular decomposition of the charge content within the muffin-tin radius for the $\mathrm{Ce}$ and Te sites is listed in Table II, for the three supercell calculations in which the occupation number of the central cerium site [labeled $\mathrm{Ce}(1)]$ is 0,1 , and 2, respectively. Also listed in Table II is the total charge in the interstitial. Examination of the charge decomposition in Table II shows an approximate $f \rightarrow d$ transition, i.e., most of the $d$ electron shows up on the central site after the removal (addition) of an $f$ electron from (to) the central site, while the surrounding atoms are nearly unchanged. This result shows that the screening (antiscreening) of the $f$ hole is local, in agreement with similar calculations for the cerium monopnictides. ${ }^{34}$

A pertinent quantity, describing the energy dependence of the coupling of the quasilocalized crystal-field state $M=\left\{\Gamma_{j}\right\}$ with the conduction electrons is ${ }^{38,39}$

$$
V_{M}(\varepsilon) \delta_{M M^{\prime}} \equiv \sum_{n, \mathbf{k}} V_{n \mathbf{k}, M} V_{n \mathbf{k}, M}^{*} \delta\left(\varepsilon-\varepsilon_{n \mathbf{k}}\right)
$$

In the various applications, ${ }^{38} V_{M}(\varepsilon)$ is replaced by a $\left\{\Gamma_{j}\right\}$-independent average, modeled by a semielliptical or constant function of energy. The $a b$ initio calculated 
TABLE II. Supercell LMTO results for the angular-momentum charge decompositions within the muffin-tin spheres and the interstitial for CeTe, for three configurations $\left(f^{0}, f^{1}, f^{2}\right)$ of the central Ce site $\mathrm{Ce}(1) ; \mathrm{Ce}(2)$ are the other three equivalent $\mathrm{Ce}$ sites, $\mathrm{Te}(1)$ are the three equivalent $\mathrm{Te}$ sites that border $\mathrm{Ce}(1)$, and $\mathrm{Te}(2)$ is the other inequivalent $\mathrm{Te}$ site. The $4 f$ states are treated as core states.

\begin{tabular}{lcccc}
\hline \hline & & $f^{0}$ & $f^{1}$ & $f^{2}$ \\
\hline \hline $\operatorname{Ce}(1)$ & $s$ & 0.14 & 0.13 & 0.13 \\
& $p$ & 6.01 & 5.97 & 5.90 \\
& $d$ & 2.03 & 1.16 & 0.65 \\
$\mathrm{Ce}(2)$ & $s$ & 0.13 & 0.13 & 0.15 \\
& $p$ & 5.96 & 5.97 & 5.97 \\
$\mathrm{Te}(1)$ & $d$ & 1.14 & 1.15 & 1.10 \\
& $s$ & 1.73 & 1.73 & 1.73 \\
& $p$ & 3.15 & 3.15 & 3.13 \\
$\mathrm{Te}(2)$ & $d$ & 0.13 & 0.12 & 0.10 \\
& $s$ & 1.73 & 1.73 & 1.73 \\
& $p$ & 3.15 & 3.15 & 3.14 \\
Interstitial & $d$ & 0.12 & 0.12 & 0.11 \\
\hline \hline
\end{tabular}

$V_{M}(\varepsilon)$ for the $\Gamma_{7}$ and $\Gamma_{8}$ crystal-field states of CeTe and $\mathrm{CeSb}$ is plotted as function of energy in Figs. 5 and 6, respectively. It is clear from these figures that an energyindependent $V$ is a rather poor assumption when performing quantitative calculations and when comparing theory and experiment. The energy dependence of $V_{M}(\varepsilon)$ is rich in structure, having a shape similar to that of the density of states. Note that the hybridization of the $\Gamma_{8}$ crystal-field state with band electrons is larger than that of the $\Gamma_{7}$ crystal-field state over the entire energy range of interest.

\section{Hybridization-induced crystal-field splitting}

The hybridization of band electrons with the crystalfield state $M$ results in a shift of the crystal-field state $\delta E_{M}$ given by Eq. (1). Thus, the "bare" (without hybridization dressing) crystal-field splitting, $\Delta_{\mathrm{CF}}=E\left(\Gamma_{8}\right)$

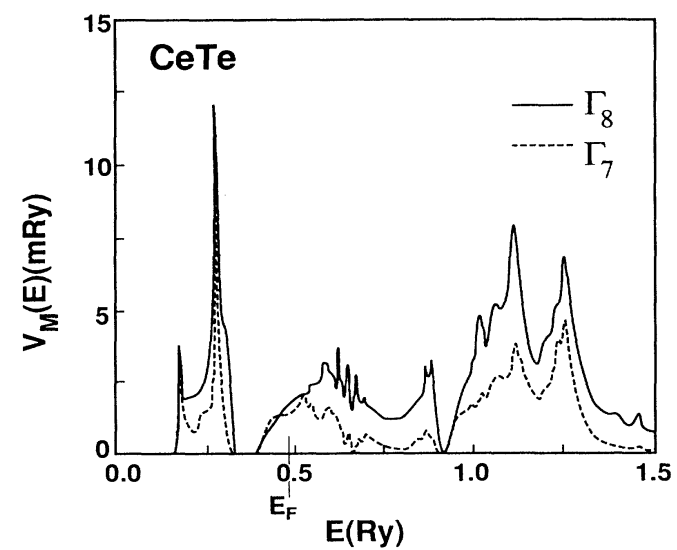

FIG. 5. The hybridization function $V_{M}(\varepsilon)$, defined in Eq. (13), between the band states and the $\Gamma_{7}$ (dashed curve) and the $\Gamma_{8}$ (solid curve) crystal-field states, respectively, in CeTe.
$-E\left(\Gamma_{7}\right)$, will be changed by an amount, $\delta \Delta_{\mathrm{CF}}=\delta E\left(\Gamma_{8}\right)-\delta E\left(\Gamma_{7}\right)$. (The experimentally observed hybridization-dressed crystal-field splitting is $\Delta_{\mathrm{CF}}^{*}=\Delta_{\mathrm{CF}}+\delta \Delta_{\mathrm{CF}}$. ) For both $\mathrm{CeSb}$ and CeTe, the hybridization-induced shift, $\delta E\left(\Gamma_{8}\right)$, of the bare crystalfield energy level of the $\Gamma_{8}$ quartet is found to be larger than the corresponding shift, $\delta E\left(\Gamma_{7}\right)$, of the $\Gamma_{7}$ doublet. This results from a combination of two effects. First, is the fact that the set of degenerate band states belonging to the star of a general point in the irreducible wedge of the Brillouin zone form a basis for a representation of the cubic group that contains the $\Gamma_{7}$ representation twice and the $\Gamma_{8}$ representation four times; thus, in a sufficiently large range of energy, there are twice as many $\Gamma_{8}$ bands to hybridize with. Second, the positive contributions to $\delta E\left(\Gamma_{7}\right)$ and $\delta E\left(\Gamma_{8}\right)$ resulting from hybridization of band states below $E_{F}$ with the $f^{2}$ state, are about equal to each

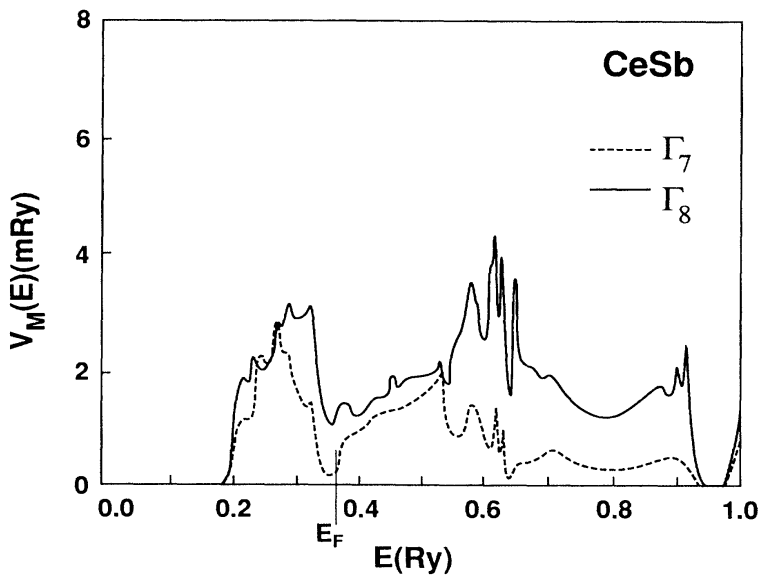

FIG. 6. The hybridization function $V_{M}(\varepsilon)$, defined in Eq. (13), between the band states and the $\Gamma_{7}$ (dashed curve) and the $\Gamma_{8}$ (solid curve) crystal-field states, respectively, in CeSb. 
other in both compounds. On the other hand, the dominant negative contribution to $\delta E\left(\Gamma_{7}\right)$ and $\delta E\left(\Gamma_{8}\right)$ resulting from hybridization of band states above $E_{F}$ with the $f^{1}$ state decreases by about $25 \%$ on going from $\mathrm{CeSb}$ to $\mathrm{CeTe}$. This arises from the fact that the hole band states in $\mathrm{CeSb}$ in the vicinity of the Fermi energy, which were yielding the largest negative contribution to $\delta E$, become occupied on going to $\mathrm{CeTe}$, thus giving a positive contribution, which is, however, reduced by a factor of $1 / U$. Furthermore, the large cancellation between the positive and negative term, which are of comparable magnitude, results in a net shift, $\delta \Delta_{\mathrm{CF}}=102 \mathrm{~K}$, in CeTe compared to the corresponding value ${ }^{23}$ of $194 \mathrm{~K}$ in CeSb. However, in the absence of a value for the bare crystal-field splitting for $\mathrm{CeTe}$, it is rather difficult to make a direct comparison of theory with experiment ${ }^{8}$ as in the case of $\mathrm{CeSb}$, where the agreement was found to be excellent. ${ }^{23}$ Including the effects of the full anisotropy of the potential within the muffin-tin spheres, which is currently under investigation, will yield an $a b$ initio value for the bare crystal-field splitting and may also affect $\delta \Delta_{\mathrm{CF}}$.

\section{Hybridization-induced two-ion coupling and magnetic behavior}

We have also calculated the $6 \times 6$ range function matrix, $\mathscr{E}_{n}=\mathscr{E}\left(m, m^{\prime}, \mathbf{R}\right) \equiv E\left(m^{\prime}, m ; m, m^{\prime} ; \mathbf{R}\right)$. This is pertinent to the great change in the magnetic behavior on going from $\mathrm{CeSb}$ to $\mathrm{CeTe}$. We find that, as in the case of $\mathrm{CeSb}$, the dominant matrix elements involve the $m, m^{\prime}= \pm 1 / 2$ states, which correspond to the piling up of charge along the interionic axis. These components for the first three nearest-neighbor shells are listed and compared for $\mathrm{CeSb}$ and $\mathrm{CeTe}$ in Table III. $\mathscr{E}_{1}$, which in the phenomenological treatment, ${ }^{2,15-22}$ is chosen to match the Néel temperature, decreases from 0.51 in $\mathrm{CeSb}$ to $0.36 \mathrm{~K}$ in CeTe. More importantly, we find that the second nearest-neighbor exchange parameter $\mathscr{E}_{2}$, which is ferromagnetic and large $(1.47 \mathrm{~K})$ in $\mathrm{CeSb}$, is antiferromagnetic and comparable in strength $(-0.180 \mathrm{~K})$ to $\mathscr{E}_{1}$ in CeTe; and $\mathscr{E}_{3}$, which is antiferromagnetic and small in $\mathrm{CeSb}$, is ferromagnetic in CeTe. This occurrence of ferromagnetic and antiferromagnetic interactions of comparable strength, and the consequent need for "compromise," is responsible for the change in magnetic behavior on going from $\mathrm{CeSb}$ to $\mathrm{CeTe}$. To illustrate further the physical basis for the difference in the unusual magnetic behavior in the two compounds, in Figs. 7 and 8 we have plotted the variation of the $a b$ initio diagonal matrix elements of $\mathfrak{B}$ [calculated from Eq. (4)] with the angle $\theta$ between the interionic axis and the axis of magnetic order-

TABLE III. Values of the hybridization-mediated two-ion exchange parameters, $\mathscr{E}_{n}=\mathscr{E}\left(m= \pm 1 / 2, m^{\prime}= \pm 1 / 2, \mathbf{R}_{n}\right)$, for the three nearest-neighbor shells $(n=1,2,3)$ for CeSb and CeTe.

\begin{tabular}{lllr}
\hline \hline & & $\mathrm{CeSb}$ & \multicolumn{1}{c}{ CeTe } \\
\hline $\mathscr{E}_{1}$ & $(\mathbf{K})$ & 0.508 & 0.365 \\
$\mathscr{E}_{2}$ & $(\mathbf{K})$ & 1.471 & -0.180 \\
$\mathscr{E}_{3}$ & $(\mathbf{K})$ & -0.0005 & 0.021 \\
\hline \hline
\end{tabular}

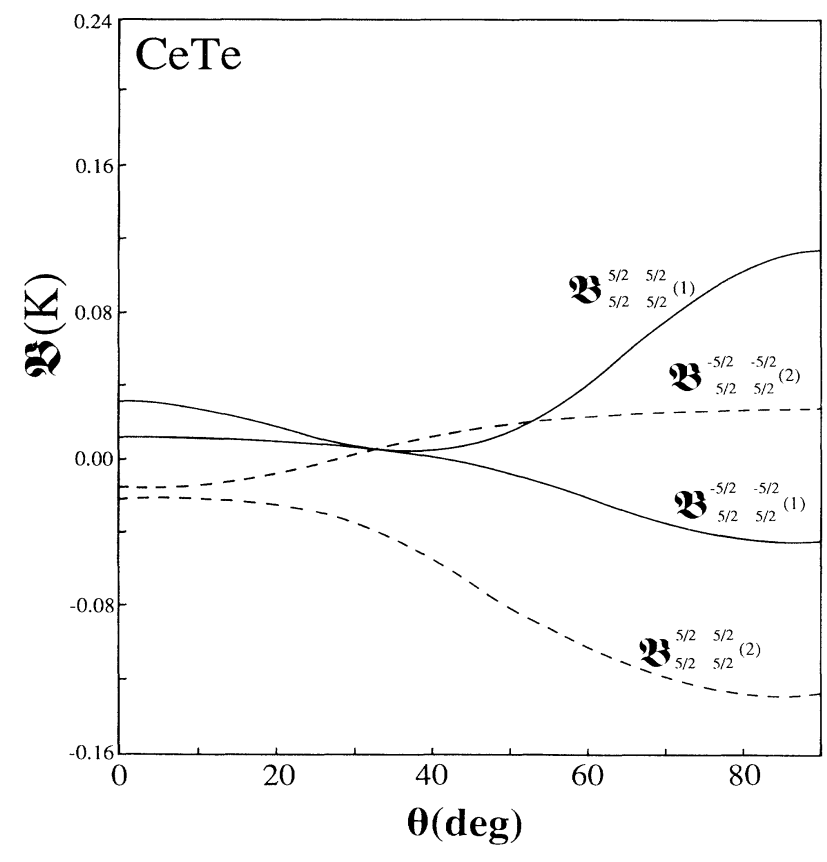

FIG. 7. Diagonal matrix elements of $\mathfrak{B}$ [calculated from Eq. (4)] for coupling between first- and second-nearest-neighbor $\mathrm{Ce}^{3+}$ ions with saturated parallel and antiparallel moments plotted vs the angle $\theta$ between the interionic axis and the axis of magnetic ordering in CeTe. The upper indices label the $M_{J}$ states on one $\mathrm{Ce}^{3+}$ ion, and the lower indices label the states of the other ion.

ing. This has been done for the first and second nearestneighbor $\mathrm{Ce}^{3+}$ ions in $\mathrm{CeTe}$ and $\mathrm{CeSb}$, respectively. It is important to point out that in our previous phenomenological treatment ${ }^{2,15-22}$ of hybridization-induced magnetism in cerium compounds, the angular dependence of $\mathfrak{B}$ was the same, apart from an overall scale factor $E_{n}$ in Eq. (5), for every symmetry shell, whereas the angular dependence of the $a b$ initio calculated $\mathfrak{B}$ depends also upon the symmetry shell. The change in magnetic behavior can be understood by detailed examination of the matrix elements giving the anisotropy of the hybridizationmediated two-ion exchange interactions. The matrix elements $\mathfrak{B}_{5 / 2}^{5 / 2} 5 / 2$ and $\mathfrak{B}_{5 / 2}^{-5 / 2} \frac{-5 / 2}{5 / 2}$ characterize the anisotropy of the exchange coupling for a pair of $\mathrm{Ce}^{3+}$ ions, when both have saturated moments, which are parallel and antiparallel, respectively. In $\mathrm{CeSb}$, the positive value of $\mathfrak{B}_{5 / 2}^{5 / 25 / 2}$ and the negative value of $\mathfrak{B}_{5 / 2}^{-5 / 2} \frac{-5 / 2}{5 / 2}$ for both the first- and second-nearest neighbors at $\theta=\pi / 2$, corresponds to strong ferromagnetic coupling within (001) planes and weak coupling between planes. On the other hand, in CeTe, $B_{5 / 2}^{5 / 2} 5 / 2$ for a nearest-neighbor $\mathrm{Ce}^{3+}$ pair peaks at $\theta=\pi / 2$, while $B_{5 / 2}^{-5 / 2} \frac{-5 / 2}{5 / 2}$ for a second-nearestneighbor pair has a negative value at $\theta=\pi / 2$, favoring antiferromagnetic moment alignment within the (001) planes. This competition of ferromagnetic and antiferromagnetic coupling within the (001) planes in turn changes the polarization from the [001] to the [111] direction.

Upon treating the two-ion Hamiltonian of Eq. (3) within mean-field theory and using the $a b$ initio calculat- 


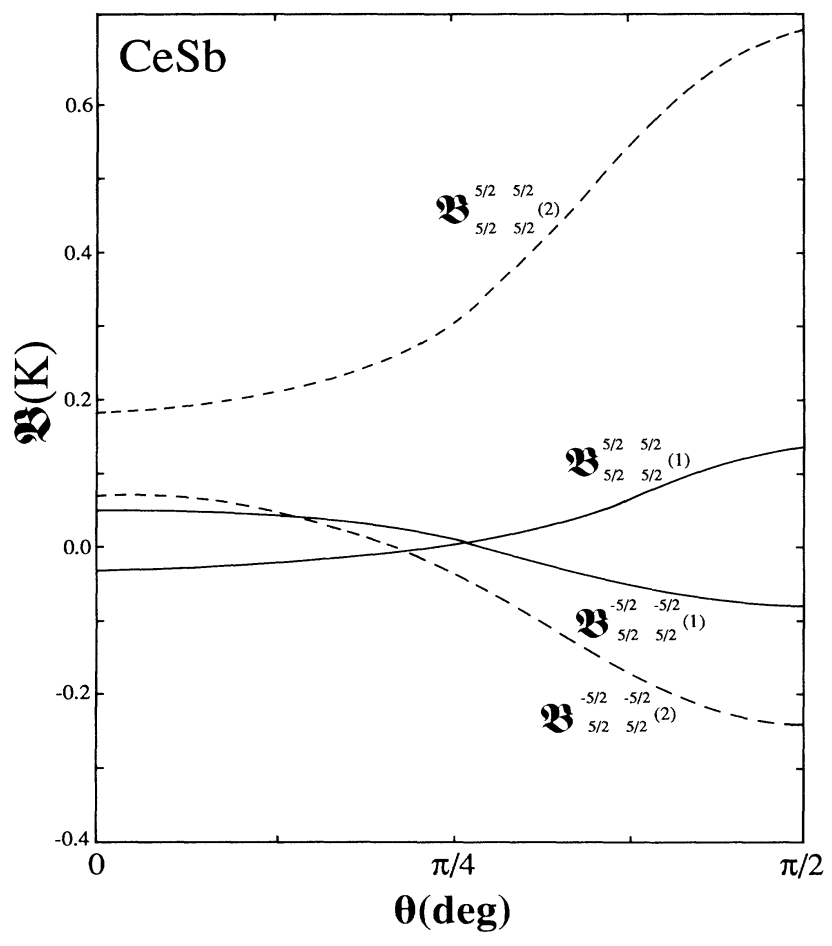

FIG. 8. Diagonal matrix elements of $\mathfrak{B}$ [calculated from Eq. (4)] for coupling between first- and second-nearest-neighbor $\mathrm{Ce}^{3+}$ ions with saturated parallel and antiparallel moments plotted vs the angle $\theta$ between the interionic axis and the axis of magnetic ordering in CeSb. The upper indices label the $M_{J}$ states on one $\mathrm{Ce}^{3+}$ ion, and the lower indices label the states of the other ion.

ed two-ion exchange interaction matrix, $E\left(m_{1}, m_{1}^{\prime} ; m_{2}, m_{2}^{\prime} ; \mathbf{R}\right)$, we have also investigated the change in equilibrium magnetic behavior on going from $\mathrm{CeSb}$ to CeTe. If we treat the two-ion exchange matrix at the highest level of approximation, including the large ionic separation limit, as described in Sec. II, there is only one two-ion exchange parameter per neighbor shell $\mathscr{E}_{n}$ (the predominant components are those for which $m_{1}, m_{1}^{\prime}= \pm 1 / 2$, with all others being about one order of magnitude smaller). Values of these are listed in Table III. At this level of approximation, we find that CeSb orders at $T_{c}=1.7 \mathrm{~K}$ into a $\{001\}$-type ferromagnetic structure with a zero-temperature free energy of $-2.6 \mathrm{~K}$ per cerium ion, whereas CeTe orders at $T_{c}=0.36 \mathrm{~K}$ into a \{111\}-type ferromagnetic structure with a zerotemperature free energy of $-0.6 \mathrm{~K}$ per cerium ion. This difference in the decrease in the free energy associated with magnetic ordering indicates that $\mathrm{CeSb}$ is a well magnetically ordered system, while CeTe is only weakly ordered. Indeed, the calculations give a low-temperatureordered Ce moment of $1.7 \mu_{B}$ close to saturation $\left(2.1 \mu_{B}\right)$ for $\mathrm{CeSb}$, predict a ratio of Néel temperatures of about 5 , and also predict the correct change in direction of ordered moment, in qualitative agreement with experiment. The degree of approximation of the two-ion exchange matrix can be improved by eliminating the large ionic separation limit approximation, i.e., level of treatment where one includes the full $6 \times 6 a b$ initio calculated exchange parameters per neighbor shell. On doing this, the zero-temperature free energy per cerium ion decreases further in $\mathrm{CeSb}$ to $-3.8 \mathrm{~K}$, the low-temperature magnetic moment increases further to $2.1 \mu_{B}$, and the Néel temperature increases further to $2.4 \mathrm{~K}$, indicating that the well-ordered state of CeSb is "confirmed" by improving the degree of approximation. On the other hand, for $\mathrm{CeTe}$, there is now no stable magnetically ordered state at all temperatures (the free energy of the cerium ion has been raised close to zero). Thus, the increase in band- $f$ hybridization places $\mathrm{CeTe}$ at the borderline between magnetic and nonmagnetic behavior in contrast to the almost fully ordered CeSb. However, although the hybridization-induced exchange interactions give the correct trend in magnetic behavior, their overall magnitude is about a factor of 6 smaller than that required to yield Néel temperatures which agree with experiment. ${ }^{1,3,4}$ By including both the hybridization-induced exchange interactions and the Ruderman-Kittel-Kasuya-Yosida (RKKY) interactions on an equal footing, Sheng and Cooper have recently extended ${ }^{40}$ the phenomenological Hamiltonian. While the inclusion of the RKKY interactions (which are also evaluated $a b$ initio) yields a two-ion interaction whose range and angular dependence is quite similar to the hybridization-induced interaction, the scale of energy increases by an order of magnitude, thus yielding Néel temperatures in good agreement with experiment.

\section{NONLINEAR HYBRIDIZATION EFFECTS}

In calculating the crystal-field splitting in Sec. IV, the band energies entering Eq. (1) were obtained from a LMTO band-structure calculation, which, as described in Sec. III, neglects band- $f$ hybridization, i.e., from a calculation that is linear in hybridization. Including such hybridization effects will change the occupation of the band states and $f$ states near the Fermi energy, and this in turn will alter the hybridization itself, thus introducing nonlinear hybridization effects. In this section we investigate whether inclusion of the nonlinear hybridization effects associated with the self-consistent determination of both the band states and the $f$ states in the presence of the band- $f$ hybridization are apt to be important in determining the crystal-field splitting.

The direct scattering term arising from the SchriefferWolff $^{28}$ transformation when applied to the Anderson Hamiltonian gives rise $\mathrm{e}^{23,27}$ to a hybridization-induced shift of the band states of the form,

$$
\begin{aligned}
\delta \varepsilon_{\mathbf{k}}= & -\theta\left(E_{F}-\varepsilon_{\mathbf{k}}\right) \sum_{M}\left\langle n_{M}\right\rangle\langle J(\mathbf{k}, M, 1 ; \mathbf{k}, M, 1) \\
& \left.+\sum_{M^{\prime} \neq M} J\left(\mathbf{k}, M^{\prime}, 1 ; \mathbf{k}, M^{\prime}, 1\right)\right] \\
& +\theta\left(\varepsilon_{\mathbf{k}}-E_{F}\right) \sum_{M}\left\langle n_{M}\right\rangle J(\mathbf{k}, M, 0 ; \mathbf{k}, M, 0) .
\end{aligned}
$$

Here, $M$ is the crystal-field state, $\left\langle n_{M}\right\rangle$ is the average occupation number of $|M\rangle$, and $J(\mathbf{k}, M, n ; \mathbf{k}, M, n)$ is given by Eq. (2). The first term in Eq. (14), which is negative, 
represents the hybridization-induced energy shift of an electron, and the second term, which is positive, represents the corresponding shift for a hole. The energy shift is larger for those band states which are close to the Fermi energy (typically of about 4-10 mRy). Consequently, the nonlinear hybridization effects open a small energy gap around the Fermi energy. Note that the hybridization-induced energy shift of a band state depends on the type of the crystal-field ground state, and on the thermal occupation of the excited crystal-field state. Thus the energy bands, in the presence of nonlinear hybridization effects, are solutions of the self-consistent equation

$$
\varepsilon_{\mathrm{k}}=\varepsilon_{\mathrm{k}}^{(0)}+\delta \varepsilon_{\mathrm{k}}
$$

where $\varepsilon_{\mathrm{k}}^{(0)}$ are the band energies in the absence of band- $f$ hybridization. Note that Eq. (15) is quadratic in the $\varepsilon_{\mathbf{k}}$.

We have investigated the nonlinear hybridization effects on the crystal-field splitting, by calculating first the band energies $\varepsilon_{\mathbf{k}}^{(0)}$ in the absence of band- $f$ hybridization (as described in Sec. III), then calculating the hybridization matrix elements $V_{\mathrm{k} M}$ from Eq. (7) and the band energies $\varepsilon_{\mathrm{k}}$ in the presence of nonlinear hybridization effects from Eqs. (14) and (15), respectively, and finally recalculating a shift of the crystal-field state $\delta E_{M}$ from Eq. (1). We find the nonlinear hybridization effects on $\delta \Delta_{\mathrm{CF}}$ to be very small $(0.5 \mathrm{~K})$ for both $\mathrm{CeSb}$ and $\mathrm{CeTe}$. This is due to the fact that there are only very few band energies close to $E_{F}$ in the tetrahedral mesh $^{35}$ used to evaluate $\delta E_{M}$ in Eq. (1), and $V_{\mathrm{k} M}$ is only of the order of a few mRy. We have also investigated the importance of nonlinear hybridization effects on the self-consistent oneelectron potential describing the band structure of each compound, by using the band states in the presence of nonlinear hybridization effects $\varepsilon_{\mathbf{k}}$ rather than $\varepsilon_{\mathbf{k}}^{(0)}$, to construct the potential in the self-consistent process, and found them to be small (less than $1 \mathrm{mRy}$ ). Thus, contrary to the tight-binding results of Takahashi and Kasuya, ${ }^{26}$ we find that nonlinear hybridization effects are not important to the hybridization-induced suppression of the crystal-field splitting in $\mathrm{CeSb}$ and $\mathrm{CeTe}$.

\section{CONCLUSION}

We have applied a theory, which involves a synthesis of (1) a phenomenological theory of orbitally driven magnetic ordering of moderately delocalized $f$ electron systems based on the Anderson-lattice Hamiltonian and (2) $a b$ initio electronic-structure calculations allowing a first-principles evaluation of the parameters entering the phenomenological theory, to investigate, in terms of the electronic structure, the change in magnetic behavior on going from the well-ordered $\mathrm{CeSb}$ to $\mathrm{CeTe}$, which is at the borderline between magnetic and nonmagnetic behavior. The addition of an anion $p$ electron causes the Fermi energy to rise into a region of high cerium $5 d$-derived density of states, and causes the anion $p$-derived bands to sink far below the Fermi energy. Consequently, the $p-f$ hybridization is suppressed, and the bands dominating the hybridization-induced properties in CeTe are largely derived from the Ce $5 d$ states. The increase of the reso- nance width $\Gamma$ from $2.96 \mathrm{mRy}$ in CeSb to $5.67 \mathrm{mRy}$ in $\mathrm{CeTe}$, and the resultant increase of the hybridization potential $v\left(\kappa_{F}\right)$ from $2.33 \mathrm{mRy}$ to $3.08 \mathrm{mRy}$, respectively, further illustrates the trend of the band- $f$ hybridization between the two compounds. In both systems, the $4 f^{1}$ state is found to be about $3.0 \mathrm{eV}$ below the Fermi energy, in agreement with photoemission experiments, ${ }^{36}$ and the intra-atomic Coulomb interaction $U$ is found to be about $6.0 \mathrm{eV}$. The calculated hybridization-induced suppression of the crystal-field splitting, $\delta \Delta_{\mathrm{CF}}$, decreases from $194 \mathrm{~K}$ in $\mathrm{CeSb}$ to $102 \mathrm{~K}$ in CeTe. However, in the absence of a value for the bare crystal-field splitting for $\mathrm{CeTe}$, it is rather difficult to make a direct comparison with experiment ${ }^{8}$ as in the case of $\mathrm{CeSb}$, where the agreement was found to be excellent. ${ }^{23}$ Including the effects of the full potential within the muffin-tin spheres, which is currently under investigation, will yield an $a b$ initio value for the bare crystal-field splitting and may also affect $\delta \Delta_{\mathrm{CF}}$. Nonlinear hybridization effects, associated with the self-consistent determination of both the band states and $f$ states in the presence of band- $f$ hybridization, on the hybridization-dressing of the crystal-field are found to be small in both systems. The results of the calculations illustrate the sensitivity of the hybridization-induced exchange interactions to chemical environment and to the details of the electronic structure. We find that the striking change of the magnitude, sign, and the range dependence of the hybridization-induced two-ion exchange interactions place CeTe at the borderline between magnetic and nonmagnetic behavior in contrast to the almost fully ordered $\mathrm{CeSb}$. However, the overall magnitude of the hybridization-induced exchange interactions is about a factor of 6 smaller than that required to yield critical temperatures which agree with experiment., ${ }^{1,4}$ By including both the hybridization-induced exchange interactions and the RKKY interactions (which are neglected in this work) on an equal footing, Sheng and Cooper have recently extended ${ }^{40}$ the phenomenological model Hamiltonian. While the inclusion of the RKKY interactions yields a two-ion interaction whose range and angular dependence is quite similar to the hybridization-induced effects, the scale of the interaction is found to increase by an order of magnitude, thus yielding ordering temperatures in good agreement with experiment. ${ }^{40}$ On the other hand, the inclusion of the RKKY interactions will not affect the hybridization-induced suppression of the crystal-field splitting, which is purely a hybridizationinduced effect. ${ }^{40}$

\section{ACKNOWLEDGMENTS}

The research at California State University Northridge (CSUN) was supported through the National Science Foundation under Grant No. DMR-89-18887, the Research Corporation under Grant No. C-2707, and the Office of Research and Sponsored Projects at CSUN. The research at West Virginia University was supported through the National Science Foundation under Grant No. DMR-88-07523 and at Los Alamos National Laboratory by the U.S. Department of Energy. 
1J. Rossat-Mignod, P. Burlet, S. Quezel, J. M. Effantin, D. Delacôte, H. Bartholin, O. Vogt, and D. Ravot, J. Magn. Magn. Mater. 31-34, 398 (1983).

${ }^{2}$ B. R. Cooper, R. Siemann, D. Yang, P. Thayamballi, and A. Banerjea, in The Handbook of the Physics and Chemistry of the Actinides, edited by A. J. Freeman and G. H. Lander (North-Holland, Amsterdam, 1985), Vol. 2, Chap. 6, pp. 435-500.

${ }^{3}$ F. Hulliger, B. Natterer, and H. R. Ott, J. Magn. Magn. Mater. 8, 87 (1978).

${ }^{4}$ H. R. Ott, J. K. Kjems, and F. Hulliger, Phys. Rev. Lett. 42, 1378 (1979).

${ }^{5}$ G. Busch and O. Vogt, Phys. Lett. 25A, 449 (1967).

${ }^{6}$ R. J. Birgenau, E. Bucher, J. P. Maita, L. Passell, and K. C. Turberfield, Phys. Rev. B 8, 5345 (1973).

${ }^{7}$ J. Rossat-Mignod, J. M. Effantin, C. Vettier, and O. Vogt, Physica B 130, 555 (1985); J. Rossat-Mignod, J. D. Delacôte, J. M. Effantin, C. Vettier, and O. Vogt, ibid. 120, 163 (1983).

${ }^{8}$ J. Rossat-Mignod, J. M. Effantin, P. Burlet, T. Chattopadhyay, L. P. Regnault, H. Bartholin, C. Vettier, O. Vogt, D. Ravot, and J. C. Achart, J. Magn. Magn. Mater. 52, 111 (1985).

${ }^{9}$ B. R. Cooper and O. Vogt, J. Phys. (Paris) Colloq. 32, C1-1026 (1971).

${ }^{10}$ H. Bartholin, O. Vogt, and J. P. Senateur, J. Magn. Magn. Mater. 15-18, 1247 (1980).

${ }^{11}$ D. Ravot, A. Mauger, J. C. Achard, M. Bartholin, and J. Rossat-Mignod, Phys. Rev. B 28, 4558 (1983).

${ }^{12}$ J. Rossat-Mignod, P. Burlet, J. Villain, H. Bartholin, T. S. Wang, D. Florence, and O. Vogt, Phys. Rev. B 16, 440 (1977).

${ }^{13}$ D. Ravot, P. Burlet, J. Rossat-Mignod, and J. L. Tholence, J. Phys. (Paris) Colloq. 41, C1-1117 (1980).

${ }^{14}$ M. Escorne, D. Ravot, J. C. Achard, and A. Mauger, J. Appl. Phys. 53, 2061 (1982).

${ }^{15}$ R. Siemann and B. R. Cooper, Phys. Rev. Lett. 44, 1015 (1980).

${ }^{16}$ N. Kioussis and B. R. Cooper, Phys. Rev. B 34, 3261 (1986).

${ }^{17}$ G.-J. Hu and B. R. Cooper, Phys. Rev. B 38, 9127 (1988).

${ }^{18}$ N. Kioussis, B. R. Cooper, and A. Banerjea, Phys. Rev. B 38, 9132 (1988)

${ }^{19}$ A. Banerjea and B. R. Cooper, Phys. Rev. B 34, 1607 (1986).
${ }^{20}$ B. R. Cooper, G.-J. Hu, N. Kioussis, and J. M. Wills, J. Magn. Magn. Mater. 63-64, 121 (1987); B. R. Cooper, J. LessCommon Met. 133, 31 (1987).

${ }^{21}$ G.-J. Hu, N. Kioussis, A. Banerjea, and B. R. Cooper, Phys. Rev. B 38, 2639 (1988).

${ }^{22}$ G.-J. Hu and B. R. Cooper, J. Appl. Phys. 64, 5592 (1989); G.-J. Hu, B. R. Cooper, and G. H. Lander, Physica B 156-157, 822 (1989).

${ }^{23}$ J. M. Wills and B. R. Cooper, Phys. Rev. B 36, 3809 (1987).

${ }^{24}$ B. R. Cooper, J. M. Wills, N. Kioussis, and Q.-G. Sheng, J. Appl. Phys. 64, 5587 (1988); B. R. Cooper, J. M. Wills, N. Kioussis, and Q.-G. Sheng, J. Phys. (Paris) Colloq. 49, C8463 (1988)

${ }^{25}$ Q.-G. Sheng, B. R. Cooper, J. M. Wills, and N. Kioussis, J. Appl. Phys. 67, 5197 (1990).

${ }^{26}$ H. Takahashi and T. Kasuya, J. Phys. C 18, 2697 (1985).

${ }^{27}$ B. Coqblin and J. R. Schrieffer, Phys. Rev. 185, 847 (1969); B. Cornut and B. Coqblin, Phys. Rev. B 5, 4541 (1972).

${ }^{28}$ J. R. Schrieffer and P. A. Wolff, Phys. Rev. 149, 491 (1966); J. R. Schrieffer, J. Appl. Phys. 38, 1143 (1967).

${ }^{29}$ S. B. Haley and P. Erdos, Phys. Rev. B 5, 1106 (1972).

${ }^{30}$ A. R. Edmonds, Angular Momentum in Quantum Mechanics (Princeton University Press, New Jersey, 1974).

${ }^{31}$ H. L. Skriver, The LMTO Method (Springer, Berlin, 1984); O. K. Andersen, Phys. Rev. B 12, 3060 (1975).

${ }^{32}$ L. Hedin and B. I. Lundqvist, J. Phys. C 4, 2064 (1971).

${ }^{33}$ D. D. Koelling and B. N. Harmon, J. Phys. C 10, 3107 (1977).

${ }^{34}$ M. R. Norman, D. D. Koelling, A. J. Freeman, H. J. F. Jansen, B. I. Min, T. Oguchi, and Ling Ye, Phys. Rev. Lett. 53, 1673 (1984); M. R. Norman, D. D. Koelling, and A. J. Freeman, Phys. Rev. B 31, 6251 (1985).

${ }^{35}$ G. Gilat and N. R. Bharatiya, Phys. Rev. B 12, 3497 (1975).

${ }^{36} \mathrm{~W}$. Gudat, M. Campagna, R. Rosei, J. H. Weaver, W. Eberhardt, F. Hulliger, and E. Kaldis, J. Appl. Phys. 52, 2123 (1981).

${ }^{37}$ Q.-G. Sheng, B. R. Cooper, N. Kioussis, and J. M. Wills, Bull. Am. Phys. Soc. 33, 364 (1988).

${ }^{38} \mathrm{O}$. Gunnarsson and K. Schonhammer, Phys. Rev. B 28, 4315 (1983), and references therein.

${ }^{39}$ R. Monnier, L. Degiorgi, and D. D. Koelling, Phys. Rev. Lett. 56, 2744 (1986).

${ }^{40}$ Q.-G. Sheng and B. R. Cooper, J. Appl. Phys. 69, 5472 (1991). 